\title{
Les objectifs de la réforme du lycée et du baccalauréat en France
}

\section{Marie-Pierre Luigi}

\section{OpenEdition}

1 Journals

Édition électronique

URL : https://journals.openedition.org/ries/8579

DOI : $10.4000 /$ ries.8579

ISSN : 2261-4265

Éditeur

France Education international

\section{Édition imprimée}

Date de publication : 1 septembre 2019

Pagination : 15-19

ISBN : 978-2-85420-624-1

ISSN : $1254-4590$

Référence électronique

Marie-Pierre Luigi, "Les objectifs de la réforme du lycée et du baccalauréat en France », Revue internationale d'éducation de Sèvres [En ligne], 81 | septembre 2019, mis en ligne le 01 septembre 2021, consulté le 13 octobre 2021. URL : http://journals.openedition.org/ries/8579 ; DOI : https://doi.org/ 10.4000/ries.8579

Ce document a été généré automatiquement le 13 octobre 2021.

(c) Tous droits réservés 


\title{
Les objectifs de la réforme du lycée et du baccalauréat en France
}

\author{
Marie-Pierre Luigi
}

1 Le baccalauréat français est une institution de plus de deux cents ans ; hier réservé à une élite, il concerne aujourd'hui 520000 candidats en voie générale et technologique, dont les taux de réussite ont sans cesse évolué. Ainsi, ces trente dernières années, la proportion de bacheliers dans une classe d'âge est passée de 30 à $80 \%$.

2 En se démocratisant, le baccalauréat se complexifiait avec une multiplication de disciplines et d'épreuves, près de 3000 sujets, 170000 correcteurs et examinateurs, et 4 millions de copies à corriger. Parallèlement, les taux d'échecs constatés dans les premières années d'enseignement supérieur remettaient en question le baccalauréat en tant que premier diplôme de l'enseignement supérieur.

3 En 2017, le président de la République annonçait sa volonté de «simplifier son organisation et restaurer sa crédibilité ». La mission mandatée par le ministre de l'éducation nationale et pilotée par Pierre Mathiot, professeur d'université, remit son rapport en janvier 2018; elle dressait un diagnostic et proposait les lignes d'une réforme du baccalauréat et du lycée général et technologique.

4 En ressortiront principalement la suppression des séries différenciant jusqu'ici les parcours des lycéens de la voie générale : la série scientifique, la série littéraire et la série économique. Les séries de la voie technologique (tertiaire, santé, laboratoire, technologies industrielles...) ont été maintenues mais pour ces deux voies, l'organisation des enseignements connaît les mêmes évolutions. Enfin, tous les programmes d'enseignement du nouveau lycée ont été réécrits pour une mise en œuvre à la présente rentrée 2019. La première génération d'élèves concernés par cette réforme est entrée au lycée en septembre 2018.

\section{Des constats lourds de sens}

5 Les constats établis en 2017 par la mission Mathiot ${ }^{1}$ ont confirmé ce que le grand public pressentait déjà depuis longtemps : le baccalauréat, auquel accède aujourd'hui la très 
grande majorité d'une classe d'âge, perd de sa crédibilité et représente une organisation lourde et coûteuse.

6 Mais le constat le plus difficile est sans nul doute que le baccalauréat, obtenu par plus de $80 \%$ des candidats des voies technologique et générale, ne garantit pas la réussite des futurs étudiants. Plus de la moitié des inscrits en licence échouaient dans la voie qu'ils avaient choisie. Une voie d'autant plus sinueuse qu'ils s'y risquaient sans véritable anticipation et que leur choix de filière ne présageait en rien de la suite de leur parcours. Ainsi, la prédominance de la série scientifique, peu à peu réservée aux meilleurs d'entre eux, ne signifiait nullement un engouement pour ces disciplines: même si plus de la moitié des élèves de la voie générale s'y retrouvaient, près de la moitié d'entre eux n'avaient aucun projet dans ce domaine.

7 Parallèlement à ces constats, 40000 lycéens consultés par le ministère de l'éducation nationale en novembre 2017 demandaient massivement la disparition des séries de la voie générale et l'introduction d'un contrôle continu qu'ils estimaient plus juste que des épreuves finales trop nombreuses, anxiogènes, et trop éloignées de leurs progressions quotidiennes.

8 Les constats étaient lourds de sens: le baccalauréat représentait des organisations pesantes et coûteuses, apparaissait injuste, et surtout ne donnait aucune garantie de réussite dans l'enseignement supérieur.

Dès lors toute réforme du baccalauréat et du nouveau lycée devait répondre à un objectif principal : en préparant le baccalauréat, les élèves devaient ancrer leur réussite après le baccalauréat.

\section{Accompagner les apprentissages}

10 Le nouveau lycée vise à donner aux élèves les moyens de leurs ambitions et la liberté de leurs choix. C'est ainsi que leur accompagnement est renforcé, année après année, dans deux directions.

11 Le premier accompagnement des élèves est le soutien prévu pour les aider à prendre conscience de leurs acquis et de leurs besoins. L'introduction d'un test de positionnement en début de seconde en est la première étape. Ce test permet aux élèves d'évaluer leurs acquis et leurs besoins en mathématiques et maîtrise de la langue française. Il est suivi d'un soutien mis en place en fonction des besoins de chacun. Les contrôles continus s'inscrivent dans cette démarche, avec l'analyse partagée des performances de chaque élève. Le débat entre l'évaluation certificative ou formative n'a pas lieu d'être : toute évaluation doit porter en elle ces deux axes, avec un échange systématique entre famille, élève et enseignants à propos des résultats obtenus, des acquis ou des approfondissements encore nécessaires.

12 Pour gagner en maturité dans les choix qu'ils auront à faire tout au long du lycée, les élèves devront également être informés, aidés et conseillés par les enseignants, les équipes éducatives et les professionnels de l'orientation, à chaque niveau du lycée. Dans cet objectif, au-delà des enseignements, des temps spécifiques sont prévus chaque année pour l'aide à l'orientation des élèves, l'information sur l'enseignement supérieur et les métiers. C'est aussi l'occasion d'une collaboration renforcée entre tous les acteurs de l'information à l'orientation, et avec les collectivités régionales qui en ont la compétence depuis janvier 2019. 
13 Ainsi, après la décision du conseil de classe de fin de seconde s'agissant de la voie générale ou technologique souhaitée, les élèves de la voie générale feront librement leurs choix d'enseignements de spécialité en toute connaissance de cause et avec l'éclairage des recommandations du conseil de classe. Le premier effet de cette responsabilisation, constaté dès cette année, est un intérêt accru des élèves pour les programmes à venir ainsi que pour le contenu et les apports des enseignements.

\section{Les enseignements communs, axe principal du nouveau lycée}

La structuration du nouveau lycée est désormais la même dans les deux voies, générale et technologique :

- une classe de seconde repensée avec des enseignements nouveaux: un enseignement « numérique et technologie » très novateur et un enseignement de sciences économiques et sociales pour tous les élèves ;

- un cycle terminal organisé autour de trois constantes : des enseignements communs à tous les élèves de la même voie (générale ou technologique), des enseignements de spécialité et des enseignements optionnels laissés au choix éventuel des élèves ; alors que tous les élèves de la même série suivaient jusqu'ici les mêmes enseignements, la formule modulaire de ce nouveau lycée représente un changement majeur, tant dans les organisations que pour les choix des familles et des élèves.

15 Le premier effet de la réforme est bien celui des enseignements communs à tous les élèves d'une même voie dans les classes de première et de terminale : les élèves hier partagés dans les silos distincts de chaque série sont aujourd'hui rassemblés pour l'acquisition de connaissances incontournables à leur progression, quels que soient leurs choix de parcours. Le groupe classe y retrouve sa définition première, avec des enseignements communs qui représentent en voie générale plus de la moitié des horaires hebdomadaires des élèves, et repoussent les préjugés jusqu'ici prédominants s'agissant d'élèves scolarisés dans telle ou telle série.

16 Cette perte de repères est sans doute déstabilisante pour tout un chacun, alors qu'il était jusqu'ici facile d'établir des catégories, toujours basées sur la référence d'une excellence qui serait cantonnée à la voie scientifique; l'inquiétude a pu gagner les adultes, qui avaient connu le baccalauréat sous son ancienne formule. Elle a également $\mathrm{pu}$ créer quelques inquiétudes disciplinaires quant aux choix futurs des élèves s'agissant de leurs enseignements de spécialité (trois en première générale puis deux poursuivis en terminale). De leur côté, les élèves ont plébiscité le nouveau lycée qui leur offrait un choix très élargi.

Force est de constater aujourd'hui que la répartition de ces choix dans la voie générale n'a pas remis en question le poids des enseignements dans le lycée : les disciplines n'ont pas été déséquilibrées par ces nouvelles organisations et ont été choisies au moins dans les mêmes proportions. Ce sont les combinaisons à d'autres enseignements qui varient, avec la possibilité pour les élèves et leur famille d'opter parmi des parcours variés, plus personnalisés et moins monochromes. Alors que $85 \%$ des lycées proposaient les trois séries de la voie générale, plus de $90 \%$ proposent aujourd'hui au moins sept enseignements de spécialité et parmi eux "numérique et sciences informatiques", proposé dans plus de la moitié des établissements, avec plus de 2000 enseignants 
formés : un nouvel enseignement qui rebat largement les cartes des parcours et enrichit encore les profils des futurs étudiants. À la rentrée 2019, 220 combinaisons d'enseignements étaient possibles dans un établissement offrant douze spécialités. Les premières informations de rentrée l'indiquent : les plus gros lycées (quinze classes, soit environ 500 élèves) qui proposaient douze spécialités ont mis en place des organisations répondant à une demande de 70 à 80 combinaisons d'enseignements différentes.

18 L'offre de formation des établissements est évolutive; elle représente la première condition du nouveau lycée, dont l'ambition est d'être celui de tous les possibles.

\section{Le contrôle continu dédramatisé, pour un baccalauréat plus juste}

19 Le nouveau baccalauréat change profondément, avec l'introduction du contrôle continu. Dans son esprit, la mise en place du contrôle continu répond à une évaluation scolaire normale, filée tout au long du cycle terminal ; la nécessaire objectivation du contrôle continu qui est ressortie de la concertation avec les partenaires sociaux ne s'oppose pas à cette conception.

20 Trois séries d'épreuves ont été prévues (deux en première et une en terminale), exclusivement centrées sur les enseignements communs et sur l'enseignement de spécialité qui ne sera pas poursuivi en terminale. Ces évaluations filées sur le cycle permettront d'échanger avec les élèves sur leurs acquis et leurs besoins, et de faire ressortir leur montée en compétences. Ces épreuves, cumulées avec les résultats du livret scolaire, représentent désormais $40 \%$ de la note finale des candidats.

21 L'objectivation du contrôle continu, qui répond aux possibles craintes d'inéquité soulignées en 2018 dans le rapport Mathiot et reprises dans le cadre du dialogue social, induit une logistique nouvelle : l'anonymat des candidats et de leurs correcteurs, la banque nationale de sujets, les commissions académiques d'harmonisation, ne doivent pas effacer les objectifs initiaux ou dramatiser ces étapes. Les contrôles régulièrement organisés doivent permettre d'acter la progression des élèves, et s'assimilent plus à des devoirs surveillés qu'à des bacs blancs ou à des épreuves finales. C'est la condition incontournable de la réussite d'une démarche qui doit permettre aux élèves de gérer leur progression et d'échapper à un stress contre-productif.

Le contrôle continu permet de réduire de douze ${ }^{2}$ à cinq le nombre d'épreuves finales nationales : l'épreuve anticipée de français maintenue en première, et quatre épreuves en fin de cycle: la philosophie, les deux enseignements de spécialité de terminale et l'oral terminal.

Ce «grand oral», qui s'appuie sur les enseignements de spécialité suivis par le candidat, arme les élèves pour leur parcours étudiant, professionnel ou personnel. Il est aussi une démarche d'équité sociale en permettant à chaque élève d'en acquérir la capacité. L'expression orale est ainsi renforcée dans les programmes et les disciplines, au lycée comme à tous les niveaux d'enseignement scolaire. 


\section{Et après le baccalauréat...}$$
\text { évolueront pour accueillir et former au mieux des bacheliers qui auront choisi leur }
$$
parcours et accédé aux formations de leur réussite.

\section{NOTES}

1. Rapport de la mission Mathiot: Un nouveau baccalauréat pour construire le lycée des possibles, [en ligne] [https://bit.ly/2VsuQUo].

2. En moyenne : le nombre d'épreuves dépendait des séries et options des candidats.

\section{INDEX}

Index géographique : France

Mots-clés : baccalauréat, réforme de l'enseignement, lycée

Keywords : French high school diploma, educational reform, upper secondary schools

Palabras claves : título de bachillerato, reforma de la educación, escuela secundaria (segundo nivel)

\section{AUTEUR}

\section{MARIE-PIERRE LUIGI}

Marie-Pierre Luigi est entrée à l'inspection générale de l'administration de l'éducation nationale et de la recherche en 2012, après une carrière administrative recouvrant les différents contextes de l'enseignement scolaire et supérieur, en établissement scolaire, université, inspection académique et rectorat, en particulier dans les académies de Créteil et de Versailles dont elle fut secrétaire générale de 2009 à 2012. Adjointe au secrétaire général du ministère de l'éducation nationale en 2015, elle a suivi plus particulièrement les questions territoriales liées au redécoupage régional en métropole et à la création des régions académiques. Elle est missionnée depuis mars 2018 par le ministre de l'éducation nationale sur la fonction de chef de projet «baccalauréat 2021 ». Courriel : marie-pierre.luigi@education.gouv.fr 